\title{
Maternal Anthropometry and it's Relationship to Birth Weight
}

\author{
Chandana Rrishna', Prakruthi $X^{2}$, Pratha V Parmar ${ }^{3}$, Ruchika Lato ${ }^{4}$ \\ ${ }^{1}$ Assistant Professor, ${ }^{2,3,4}$ Intern, Department of Community Medicine, Sri Siddhartha Medical College, Tumkur. \\ DOI: https://doi.org/10.24321/2454.325X.201836
}

\section{Abstract}

Introduction: Nutrition plays a major role in maternal and child health and it is perhaps the most influential non-genetic factor in foetal development. Understanding the relation between maternal nutrition and birth outcomes may provide a basis for developing nutritional interventions that will improve birth outcomes and long-term quality of life. Many research projects have studied maternal anthropometric indicators as predictors of birth weight; not many of them have focused on the question as to which is the best indicator for prediction of pregnancy outcomes with greater accuracy. Hence this study was taken up to assess the relationship between specific maternal anthropometric indicator including Mid-upper arm circumference (MUAC) and pregnancy outcome. The objective of the study was to assess the relationship between maternal anthropometric indicators and birth weight, crown-heel length and new-born's head circumference.

Methods: A cross-sectional study was conducted among mothers who come for safe confinement to District Hospital, Tumkur during June - August 2018. Data was collected using pre-tested, semi structured proforma. Anthropometric measurements like weight, height \& MUAC of mother and new-borns' birthweight \& length was collected. Data was analysed using SPSS and linear regression analysis was used to assess the relationship between maternal $\&$ newborn anthropometric measures.

Results: There was a statistically significant positive correlation between: Birth-weight and maternal height ( $r=0.301)$, MUAC $(r=0.222)$ \& gestational weight-gain ( $r=0.233)$; Crown-heel length of new born and MUAC $(r=0.209)$; Head circumference and MUAC $(r=0.475)$ and gestational weight-gain $(r=0.263)$.

Conclusion: MUAC as an indicator of maternal nutritional status is having a positive correlation with birth weight, and can be used as a predictor of new-born's anthropometry.

Keywords: Anthropometry, Birth-weight, Mid-upper arm circumference, Relationship

\section{Introduction}

Nutrition plays a major role in maternal and child health and is the most influential non-genetic factor in fetal development. ${ }^{1}$ Maternal body composition, nutritional stores, diet and ability to deliver nutrients through the placenta determines nutrient availability for the fetus. ${ }^{1}$ Prenatal nutrition influences fetal growth, normal development of physiological function and gestational weight gain (GWG). ${ }^{2}$
Poor maternal nutritional status has been related to adverse birth outcomes; however, the association between maternal nutrition and birth outcome is complex and is influenced by many biologic, socioeconomic, and demographic factors, which vary widely in different populations. ${ }^{3}$ Understanding the relation between maternal nutrition and birth outcomes may provide a basis for developing nutritional interventions that will improve birth outcomes and long-term quality of life and reduce mortality, morbidity, and health-care costs.

Corresponding Author: Chandana Krishna, Department of Community Medicine, Sri Siddhartha Medical College, Tumkur.

E-mail Id: chandanakrshn07@gmail.com

Orcid Id: https://orcid.org/0000-0002-1885-4149

How to cite this article: Krishna C, Prakruthi N, Parmar PV et al. Maternal Anthropometry and it's Relationship to Birth Weight. Int J Preven Curat Comm Med 2018; 4(4): 48-53. 
World Health Organization (WHO) estimates that, 25 million low birth weight (LBW) babies are born annually worldwide and $95 \%$ occur in developing countries. ${ }^{4}$ One in three babies in India are born with moderate to severe malnutrition with birth weight of $2500 \mathrm{~g}$ or below. ${ }^{5}$ There are many anthropometric measures of antenatal mothers which affect the pregnancy outcome like pre-pregnancy weight, Height, Mid-Upper Arm Circumference (MUAC), gestational weight gain, etc.

MUAC is a good indicator of the protein reserves of a body, and a thinner arm reflects wasted lean mass, i.e. malnutrition. ${ }^{6} \mathrm{MUAC}$ is the circumference of the left upper arm, measured at the mid-point between the tip of the shoulder and the tip of the elbow (olecranon process and the acromium). ${ }^{7}$

Many research projects have studied maternal anthropometric indicators as predictors of birth weight. However, fewer of them have focused on the best indicator for the prediction of pregnancy outcomes with greater sensitivity, specificity and accuracy. ${ }^{8}$ There are differences between developed and developing countries in determining the most appropriate cut-off points for anthropometric measurements. Furthermore, research is needed to evaluate whether the combined use of one or two easily measurable anthropometric indicators can have a high predictive power for risk of adverse birth outcomes. Médecins Sans Frontières (MSF) has developed a new universal MUAC strip which is awaiting field validation. ${ }^{9}$

Hence this study was taken up among the mothers coming for delivery at District hospital Tumkur, to assess the relationship between maternal anthropometric indicators and birth weight, crown-heel length and new-born's head circumference.

\section{Objectives}

\section{The objective of the study was}

- To assess the relationship between maternal anthropometric indicators and birth weight.

- To assess the relationship between maternal anthropometric indicators and crown-heel length and new-born's head circumference.

\section{Materials \& Methods}

A cross-sectional study was conducted among mothers in post-natal ward who come for safe confinement to District Hospital, Tumkur during June - August 2018. A purposive sampling method was used.

The study was conducted after getting the IEC approval. Similarly, the permission from district surgeon of the study centre was obtained. Sample Size was $100\left[R=0.399^{8}\right]$.
All the mothers in the postnatal ward who gave informed consent, after fulfilling the inclusion \& exclusion criteria were interviewed and the data was collected using pretested; semi-structured questionnaire. Data regarding prepregnancy weight of the mother, height, body mass index (pre-pregnancy weight/height ${ }^{2}$ ), gestational weight gain, and gestational age was collected by personal interview, anthropometric examination and by evaluating their medical records. UNICEF's new Mid-Upper Arm Circumference (MUAC) Measuring Tapes were used to measure mid-upper arm circumference of the mothers. The anthropometric measurements of newborn (birth-weight, crown heel length \& head circumference) were recorded within 24 hours of delivery.

\section{Inclusion Criteria}

- $\quad$ Singleton term deliveries (38-42 weeks of gestation from last menstrual period and /or pediatric assessment of the gestational age) free of any obstetrical and medical complications known to affect fetal growth.

- The mothers who gave informed consent to participate in the study.

\section{Exclusion Criteria}

- Smokers, consumers of alcoholic beverages

- Gestational hypertension

- Gestational diabetes

- High risk pregnancies

\section{Statistical Analysis}

Data was entered in Microsoft excel and analysed using SPSS version 22. Mean and standard deviations were calculated for all variables. For the statistical analysis, bivariate and stepwise forward multivariate linear regression were conducted in SPSS program. Initially, univariate association between the dependent variables (birth weight, head circumference and crown heel length) and maternal anthropometric indicators (height, age, weight, gestational weight gain, arm circumference, pre-pregnancy weight) and gestational age as independent variables, were tested. Multivariate linear regression analysis was used to investigate the effect of the independent variables on neonatal birth weight, crown heel length and head circumference and $\mathrm{p}$-values $<0.05$ were considered statistically significant.

\section{Results}

\section{Socio-demographic Profile}

The mean age + S.D of the study subjects was $23.48 \pm 3.61$ years. Majority of the study subjects were educated till high school (58.0\%) and were home-makers (95.0\%). Majority were from rural area (77.0\%). 


\section{Anthropometric Characteristics of Study Subjects}

The anthropometric characteristics of the study population are shown in Table 1.

Table 1.Anthropometric characteristics of study subjects

\begin{tabular}{|c|c|c|}
\hline Anthropometric characteristics & Mean & Standard deviation \\
\hline \multicolumn{2}{|c|}{ Mother } \\
\hline Pre-pregnancy weight (Kg) & 49.63 & 10.54 \\
\hline Maternal height (cm) & 159.52 & 8.26 \\
\hline Quetelet's index (Kg/m²) & 18.52 & 2.39 \\
\hline Mid-upper arm circumference (cm) & 23.41 & 3.92 \\
\hline Gestational weight gain (Kg) & 9.00 & 0.35 \\
\hline Birth-weight (Kg) & 2.88 & 2.87 \\
\hline Crown heel length (cm) & 45.65 & 1.79 \\
\hline Head circumference (cm) & 33.21 & 3.14 \\
\hline Gestational age (Weeks) & 37.25 & \\
\hline
\end{tabular}

Details regarding the Delivery

$82 \%$ of study subjects had caesarean section and $18 \%$ had normal delivery. Among the study subjects, $87 \%$ had newborns who were of normal weight $(2.5-3.5 \mathrm{~kg}), 9 \%$ were low birth weight babies $(<2.5 \mathrm{~kg})$ and $4 \%$ were weighing $>3.5 \mathrm{kgs}$.

Correlation between Mothers' and New-borns' Anthropometric Measurements

Bivariate regression analysis was done to assess the correlation between maternal and new-borns' anthropometric measurements. Maternal height showed the highest correlation ( $r=0.301$ ) followed by gestational weight gain $(r=0.233)$ and mid-upper arm circumference $(r=0.222)$. Pre-pregnant Quetelet's index showed the weakest correlation ( $r=0.062$ ). Maternal height, mid-upper arm circumference and gestational weight gain had a p-value $<0.05$.

Correlation between New-borns' Crown-heel Length and Maternal Anthropometric Measurements

Maternal Mid-upper arm circumference showed the highest correlation ( $r=0.209)$ and it was statistically significant $(p<0.05)$. Weakest correlation was found for Quetelet's index $(r=0.074)$.

Correlation between New-borns' Head Circumference and Maternal Anthropometric Measurements

Maternal mid-upper arm circumference showed the highest correlation ( $r=0.475$ ) followed by gestational weight gain (0.263) and both showed a statistically significant correlation $(p<0.05)$. Age of the mother showed the weakest correlation ( $r=0.059)$.

Table 2.Correlation coefficients between mothers' and new-borns' anthropometric measurements

\begin{tabular}{|c|c|c|c|c|c|c|}
\hline \multirow{2}{*}{ Mothers' variables } & \multicolumn{6}{|c|}{ New-born variables } \\
\cline { 2 - 7 } & \multicolumn{2}{|c|}{ Birth-weight } & \multicolumn{2}{c|}{ Crown-heel length } & \multicolumn{2}{c|}{ Head circumference } \\
\hline & $\mathbf{r}$ & $\mathbf{p}$ & $\mathbf{r}$ & $\mathbf{p}$ & $\mathbf{r}$ & $\mathbf{p}$ \\
\hline Age & 0.089 & 0.377 & 0.094 & 0.354 & 0.059 & 0.559 \\
\hline Pre-pregnancy weight & 0.187 & 0.063 & 0.114 & 0.259 & 0.161 & 0.110 \\
\hline Maternal height & 0.301 & $0.002^{*}$ & 0.107 & 0.288 & 0.131 & 0.194 \\
\hline Quetelet's index & 0.062 & 0.543 & 0.074 & 0.463 & 0.103 & 0.310 \\
\hline $\begin{array}{c}\text { Mid-upper arm circumference } \\
\text { (MUAC) }\end{array}$ & 0.222 & $0.026^{*}$ & 0.209 & $0.037^{*}$ & 0.475 & $0.001^{*}$ \\
\hline Gestational weight gain & 0.233 & $0.020^{*}$ & 0.136 & 0.179 & 0.263 & $0.008^{*}$ \\
\hline Gestational age & 0.078 & 0.441 & 0.038 & 0.710 & 0.070 & 0.486 \\
\hline
\end{tabular}

*Statistically significant. 
Multiple Linear Regression Analysis: Relative Importance of the Independent Variables

The results of the multivariate linear regression models are shown in Tables 3 . In the birth-weight model, pre-pregnancy weight ( $B-2.96 p<0.05)$, maternal height ( $B 1.771 p<0.05$ ) and Quetelet's index ( $B 2.606 p<0.05$ ) continued to be predictors of birth weight. $R$ square was 0.237 which meant this model explained $23.7 \%$ of birth weight variance. In Head circumference model, MUAC ( $B 0.573 p<0.05$ ) turned out to have the statistically significant effect on head circumference. Since R square was 0218 , this model could explain $21.8 \%$ of head circumference variance.

Linear regression equation: Birth weight $=ß 0+\beta 1$ (age) $+\beta 2$ (pre-pregnancy weight) $+\beta 3$ (Height) $+\beta 4$ (Quetelet's index) $+\beta 5$ (MUAC) + $\beta 6$ (gestational weight gain) + $\beta 6$ (gestational age) .

Table 3.Linear regression of variables associated with new-born's anthropometry

\begin{tabular}{|c|c|c|c|c|c|c|c|c|c|}
\hline \multirow[t]{3}{*}{ Variables } & \multicolumn{3}{|c|}{ Birth weight } & \multicolumn{3}{|c|}{ Crown heel length } & \multicolumn{3}{|c|}{ Head circumference } \\
\hline & $\beta^{U}$ & $\beta^{s}$ & $p$ & $\beta^{U}$ & $\boldsymbol{\beta}^{\mathrm{s}}$ & $p$ & $\beta^{U}$ & $\boldsymbol{B}^{\mathrm{s}}$ & p \\
\hline & \multicolumn{3}{|c|}{$\mathrm{R}^{2}=0.237, \mathrm{p}=0.01 *$} & \multicolumn{3}{|c|}{$R^{2}=0.084, p=0.304$} & \multicolumn{3}{|c|}{$R^{2}=0.218, p<0.05^{*}$} \\
\hline Age & -0.012 & -0.129 & 0.208 & -0.121 & -0.152 & 0.174 & -0.015 & -0.030 & 0.760 \\
\hline $\begin{array}{l}\text { Pre-pregnancy } \\
\text { weight }\end{array}$ & -0.098 & -2.960 & $0.013^{*}$ & -0.224 & -0.824 & 0.520 & -0.110 & -0.647 & 0.570 \\
\hline $\begin{array}{l}\text { Maternal } \\
\text { height }\end{array}$ & 0.075 & 1.771 & $0.003^{*}$ & 0.172 & 0.494 & 0.445 & 0.068 & 0.312 & 0.588 \\
\hline $\begin{array}{l}\text { Quetelet's } \\
\text { index }\end{array}$ & 0.261 & 2.606 & $0.010^{*}$ & 0.592 & 0.721 & 0.510 & 0.195 & 0.378 & 0.698 \\
\hline MUAC & 0.022 & 0.181 & 0.159 & 0.221 & 0.223 & 0.113 & 0.356 & 0.573 & $<0.05^{*}$ \\
\hline $\begin{array}{l}\text { Gestational } \\
\text { weight gain }\end{array}$ & 0.017 & 0.188 & 0.063 & 0.062 & 0.085 & 0.440 & 0.050 & 0.108 & 0.269 \\
\hline Gestational age & 0.000 & 0.002 & 0.983 & 0.014 & 0.016 & 0.881 & 0.006 & 0.010 & 0.915 \\
\hline
\end{tabular}

$\beta^{\cup}$ Unstandardized coefficient; $\beta^{s}$ Standardised coefficient; * ${ }^{*}$ statistically significant.

Table 4.Linear regression of variables associated with new-born's anthropometry excluding maternal weight, Quetelet's index ad gestational weight gain

\begin{tabular}{|c|c|c|c|c|c|c|c|c|c|}
\hline \multirow[t]{3}{*}{ Variables } & \multicolumn{3}{|c|}{ Birth weight } & \multicolumn{3}{|c|}{ Crown heel length } & \multicolumn{3}{|c|}{ Head circumference } \\
\hline & $\beta^{U}$ & $\beta^{s}$ & $p$ & $\beta^{U}$ & $\beta^{s}$ & $p$ & $\beta^{U}$ & $\beta^{s}$ & $\mathbf{p}$ \\
\hline & \multicolumn{3}{|c|}{$\mathrm{R}^{2}=0.153, \mathrm{p}=0.03^{*}$} & \multicolumn{3}{|c|}{$R^{2}=0.074, p=0.117$} & \multicolumn{3}{|c|}{$\mathrm{R}^{2}=0.232, \mathrm{p}<0.05^{*}$} \\
\hline Age & -0.019 & -0.195 & 0.055 & -0.137 & -0.173 & 0.103 & -0.035 & -0.071 & 0.459 \\
\hline $\begin{array}{l}\text { Maternal } \\
\text { height }\end{array}$ & 0.012 & 0.291 & $0.004^{*}$ & 0.029 & 0.085 & 0.412 & 0.006 & 0.027 & 0.771 \\
\hline MUAC & 0.024 & 0.202 & $0.048 *$ & 0.232 & 0.234 & $0.028 *$ & 0.301 & 0.485 & $<0.05^{*}$ \\
\hline $\begin{array}{c}\text { Gestational } \\
\text { age }\end{array}$ & -0.004 & -0.040 & 0.681 & 0.000 & 0.000 & 0.996 & -0.019 & -0.032 & 0.723 \\
\hline
\end{tabular}

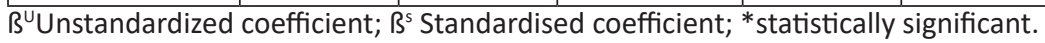

In the second model, pre-pregnancy weight, Quetelet's index and gestational weight gain was excluded to simulate late reporting in pregnancy. Results are shown in Table 4. MUAC continued to be the predictor of birth weight ( $B$ $0.202 p<0.05)$, crown heel length ( $B 0.234 p<0.05)$ and head circumference ( $B 0.485 p<0.05$ ) and was statistically significant. Maternal height also had a significant effect on birth weight ( $\$ 0.291 \mathrm{p}<0.05$ ). $R$ square indicated that $15.3 \%$ of birth weight $7.4 \%$ of crown heel length \& $23.2 \%$ of head circumference variance was explained by the 3 models (Table 4).

\section{Discussion}

Nutritional status of mothers has long been known to influence birth weight of children. Here anthropometric measurements were used as proxies for nutritional status. . MAC is easier to record and it can be used effectively in community situation even by paramedical workers. ${ }^{10}$

UNICEF supplies the MUAC tapes with both numbers and colours. It classifies mother as Severe Acute Malnutrition $(<18.5 \mathrm{~cm})$, Moderate acute malnutrition $(18.5-21 \mathrm{~cm})$, and 
at-risk mothers (21-23 cm). Management of malnutrition is based on the category: SAM: refer for intense nutritional support, MAM: refer for nutritional support, at risk: pregnant \& lactating mothers need nutritional support. ${ }^{9}$

In the present study, age of study subjects was $23.4 \pm 3$.6years, pre-pregnancy weight of $49.62 \pm 10.5 \mathrm{~kg}$, height of $159.5 \pm 8.2$ $\mathrm{cm}$, gestational weight gain of $9 \pm 3.9 \mathrm{~kg}$, gestational age of $37.24 \pm 3.14$ weeks, MUAC of $23.4 \pm 2.89 \mathrm{cms}$. In a study on maternal anthropometry as a predictor of birth weight, age of study subjects was $23.6 \pm 5.1$ years, height of $152 \pm 5.6$ $\mathrm{cm}$, MUAC of $24.2 \pm 0.2 \mathrm{cms} .{ }^{11}$ Another study on mid-upper arm circumference in pregnant women and its relation to birth weight showed maternal age of $27 \pm 6.59$ years, pre-pregnancy weight of $55.8 \pm 98 \mathrm{~kg}$, height of $156.7 \pm 5.9$ $\mathrm{cm}$, gestational weight gain of $8.8 \pm 4 \mathrm{~kg}$, gestational age of $38.08 \pm 1.32$ weeks and MUAC of $27 \pm 3.4 \mathrm{cms}^{8}$

In the present study, birth weight was $2.87 \pm 0.34 \mathrm{~kg}$, crown heel length was $45.6 \pm 2.8 \mathrm{~cm}$ and head circumference were $33.2 \pm 1.8 \mathrm{cms}$. Another study on MUAC \& birth weight showed birth weight of $3.1 \pm 0.48 \mathrm{~kg}$, crown heel length of $48.7 \pm 2.08 \mathrm{~cm}$ and head circumference of $34.4 \pm 1.4 \mathrm{~cm} .^{8}$

In the present study, low birth weight (LBW) of neonates was found to be $9 \%$. In contrast, a study in Raipur on correlation between MUAC and neonatal anthropometry, LBW was seen in $52.2 \%$ of neonates. ${ }^{12}$

In the present study, Maternal height ( $r=0.301)$, gestational weight gain ( $r=0.233$ ) and mid-upper arm circumference $(r=0.222)$ showed statistically significant correlation for birth weight. Mid-upper arm circumference showed the statistically significant correlation ( $r=0.209)$. Maternal Mid-upper arm circumference $(r=0.475)$ and gestational weight gain $(0.263)$ showed a statistically significant correlation $(p<0.05)$. Another study on MUAC and birth weight, gestational age $(r=0.425)$, MUAC $(r=0.399)$, prepregnancy weight $(r=0.378)$, Quetelet's index $(r=0.364)$ showed statistically significant correlation for birth weight. Quetelet's index ( $r=0.329)$, gestational age ( $r=0.309)$, age $(r=0.282)$, MUAC $(r=0.306)$ and pre-pregnancy weight ( $r=0.094$ ) correlated for crown heel length. ${ }^{8}$ Another study on MUAC and birth anthropometry showed correlation of 0.65 for birth weight and 0.47 for neonatal length. ${ }^{12}$

In multivariate regression, pre-pregnancy weight ( $(-2.96$, $p<0.05)$, maternal height ( $(1.771, p<0.05$ ) and Quetelet's index ( $(2.606, p<0.05)$ continued to be predictors of birth weight. MUAC ( $\$ 0.573, p<0.05)$ showed of having effect on head circumference. In a study on MUAC, multivariate regression showed that MUAC ( $ß 45.52$, $p<0.05)$ and gestational age ( $(135.48, p<0.05)$ showed to have significant effect on birth weight. ${ }^{6}$ Another study on maternal anthropometry showed that maternal height ( $B 0.273, p<0.05$ ) and pregnant BMI ( $30.398, p<0.05)$ had significant contribution to birth weight. ${ }^{11}$
On simulating late reporting of pregnancy without prepregnancy anthropometric indicators, MUAC was a significant predictor of birth weight, crown heel length and head circumference. One advantage of using MUAC to assess women at nutritional risk of giving birth to low birth weight babies is that arm circumference measurements can be taken whenever a woman visits a health worker or health centre. Recent studies have demonstrated that MUAC is closely related to maternal weight.

\section{Conclusion}

In the present study, mid upper arm circumference showed significant correlation with birth weight, crown heel length and head circumference. Additionally, maternal height \& gestational weight gain showed significant correlation with birth weight and head circumference. Multivariate linear regression showed that pre-pregnancy weight, height \& Quetelet's index were significant predictors of birth weight. On simulating late reporting of pregnancy without pre-pregnancy anthropometric indicators, MUAC was a significant predictor of birth weight, crown heel length and head circumference.

\section{Recommendations}

MUAC could be considered as a potential indicator of maternal nutritional status, and may be used instead of pre-pregnancy weight, as an alternative indicator to assess women at risk of poor pregnancy outcome in communities when presentation for prenatal care is late.

\section{Conflict of Interest: None}

\section{References}

1. Phillips DIW. External influences on the fetus and their long-term consequences. Lupus 2006; 15(11): 794-800.

2. Morgane PJ, Mokler DJ, Galler JR. Effects of prenatal protein malnutrition on the hippocampal formation. Neurosci Biobehav Rev 2002; 26: 471-83.

3. Villar J, Merialdi M, Gu“Imezoglu AM et al. Nutritional interventions during pregnancy for the prevention or treatment of maternal morbidity and preterm delivery: an overview of randomized controlled trials. J Nutr 2003; 133(5 suppl 2): 1606S-5S.

4. Sharma SR, Giri S, Timalsina $U$ et al. Low birth weight at term and its determinants in a tertiary hospital of Nepal: a case-control study. PLoS ONE 2015; 10(4). Article ID e123962.

5. Jananthan R, Wijesinghe DGNG, Sivananthawerl T. Maternal Anthropometry as a Predictor of Birth Weight. Tropical Agricultural Research 2009; 21(1): 89-98.

6. Ververs M, Antierens A, Sackl A et al. Which anthropometric indicators identify a pregnant woman as acutely malnourished and predict adverse birth outcomes in the humanitarian context? PLOS Currents 
Disasters 2013.

7. Collins S, Duffield A, Myatt M. Anthropometric assessment of the nutrition status of adults in emergency-affected populations. UN Standing Committee on Nutrition, 2000. Available from: http:// www.unscn.org/layout/modules/resources/files/ AdultsSup.pdf. Accessed on 2018 June 28.

8. Ricalde Anamaría E. Mid-upper arm circumference in pregnant women and its relation to birth weight Rev. Saúde Pública 1998; 32(2): 112-7.

9. Early detection and referral of children with malnutrition. The Mother and Child Health and Education Trust. Available from: http://motherchildnutrition.org/earlymalnutrition-detection/detection-referral-childrenwith-acute-malnutrition/interpretation-of-muac- indicators.html. Accessed on 2018 Aug 10.

10. Taksande A, Vilhekar KY, Chaturvedi P, et al. Predictor of low birth weight babies by anthropometry. Journal of Tropical Pediatrics 2007; 53(6): 420-3.

11. Winther II. Maternal anthropometry as a predictor of birth weight: a study performed at Okhaldhunga Community Hospital in rural Nepal. Master thesis, University of Oslo, 2013.

12. Rani N, Phuljhele S, Beck P. Correlation between maternal mid upper arm circumference and neonatal anthropometry. Int J Med Res Rev 2017; 5(07): 717-24.

Date of Submission: 2018-09-o6 Date of Acceptance: 2018-09-15 